\title{
Les modèles en régimes permanent et transitoire : des outils indispensables pour concevoir, optimiser la gestion et protéger les installations de production et de distribution d'eau
}

\author{
Models in steady state and transient conditions: tools for designing, management \\ optimizing and protecting water production and distribution installations
}

par J.L. Gagnon, C. Menge, F. Texier, Compagnie Générale des Eaux et J. Cavard, Syndicat des Eaux d'Ile de France

For a number of years, Water Companies have used mathematical models to simulate the behaviour of their distribution systems. At first, these tools were used to locate and design new facilities. These tools have been significantly enhanced in order to meet new requirements from project and operation managers as they are confronted with more and more complex water distribution networks and too ofien face antagonistics constraints : safety of water supplies on the one hand and quality of the distributed water on the other hand.

This paper presents, through some well selected examples, two powerful software: the Stoner Workstation Service (SWS) for analysis in steady state conditions and FLOWMASTER for transient analysis. These tools have been successfully used on the very large water distribution network of the Syndicat des Eaux d'lle de France (SEDIF) which serves approximately 4,000,000 inhabitants around Paris (France), in order to :

- resolve optimisation problems of resident time,

- determine operating strategies and pump scheduling when large diameter pipes are out of operation,

- design and optimize protection devices against waterhammer.

It is important to highlight that the accuracy and reliability of the values calculated by these models have been significantly increased to the point that these tools now allow to analyse the operation of a system in emergency and maintenance conditions and to perform sensitivity analysis.

\section{I — INTRODUCTION}

La plupart des projets d'alimentation en eau potable ne peuvent être conduits de façon totalement autonome, c'est-àdire sans tenir compte de l'influence des ouvrages existants et des modes et incidents d'exploitation. La conception est devenue aujourd'hui indissociable de l'exploitation.

Le système d'alimentation en eau potable d'un Syndicat de communes aussi important que celui du Syndicat des Eaux d'lle de France (SEDIF) se compose en effet d'un certain nombre d'installations de pompage et de stockage de l'eau dont les fonctionnements sont éminemment interdépendants. Cette interdépendance est de plus variable dans le temps et dans l'espace en fonction de l'intensité de la demande, de la configuration du réseau, de la disponibilité des installations et des modes d'asservissement. L'insertion de nouvelles installations, le renouvellement ou le renforcement de certaines conduites peut totalement bouleverser le comportement global du réseau en modifiant significativement les zones d'in- fluence de certaines installations existantes, voire en annihilant l'existence même d'un ouvrage.

La complexité croissante des systèmes d'alimentation en eau potable nécessite donc d'effectuer pour la quasi-totalité des grands projets d'aménagement ou d'extension du système d'alimentation en eau potable des études de comportement de réseau en régime hydraulique stable ou perturbé. Il en est de même pour tous travaux de voirie importants nécessitant un arrêt de "feeder" de longue durée et d'une façon générale pour toutes opérations de maintenance conduisant à une indisponibilité partielle ou totale de certains ouvrages.

\section{DEUX GRANDES FAMILLES D'ÉTUDES DE RÉSEAUX}

Il existe deux grandes familles d'études de réseaux, celle concernant les régimes dits établis ou stables, appelés de façon abusive permanents, et celle concernant les régimes dits perturbés appelés aussi transitoires. 
Lorsque, dans un réseau en régime établi survient une variation brutale de débit, causée par exemple par la fermeture d'une vanne ou l'arrêt d'une pompe, la perturbation qui en résulte génère une onde qui se propage et se réfléchit rapidement en provoquant des pics de pression positifs et négatifs dans tout le réseau. En prenant en compte la compressibilité de l'eau et l'élasticité de la conduite, on peut expliquer et modéliser la propagation et les réflexions de l'onde provoquée par ces variations brutales de débit ou de pression.

Dans les régimes stables, on suppose au contraire que l'eau est incompressible et que la conduite est rigide et on calcule alors les débits de circulation dans les conduites et les pressions en tous points du réseau en appliquant les lois bien connues de conservation de la masse et de conservation de l'énergie.

De la même façon qu'une nouvelle installation doit être conçue pour pouvoir s'intégrer dans l'environnement hydraulique existant, la détermination de sa protection anti-bélier doit procéder d'une double démarche à la fois analytique et globale.

Ces études de réseau sont menées par des experts hydrauliciens qui utilisent, pour tester la validité de leurs hypothèses, des simulateurs, c'est-à-dire des outils de calcul permettant de reproduire sur modèle le comportement du réseau réel. Bien entendu, pour pouvoir s'appuyer en toute confiance sur les résultats fournis par ces simulateurs, il s'avère indispensable de procéder au calage du modèle du réseau étudié. Cette opération de calage consiste à ajuster les paramètres du modèle jusqu'à l'obtention de la meilleure corrélation possible entre les valeurs observées lors de campagnes de mesures et les valeurs calculées par le modèle et ce, dans différentes configurations d'exploitation du réseau.

\section{III - DES MODÈLES STATIQUES AUX MODÈLES DYNAMIQUES}

Si les logiciels de calcul des réseaux maillés de distribution d'eau existent depuis près de 30 ans, la modélisation hydraulique a commencé véritablement à prendre son essor avec le développement de la micro-informatique dans les années 80 .

Des évolutions importantes de ces outils ont pu avoir lieu pour répondre aux besoins nouveaux des utilisateurs qui se trouvaient confrontés notamment à des réseaux de plus en plus complexes à étudier en termes de maillage, d'ouvrages et d'organes de régulation et à des contraintes de plus en plus fortes et souvent antagonistes en termes de sécurité (assurer les besoins en eau en toutes circonstances) et de maintien de la qualité de l'eau (limiter les temps de séjour). Essayons de retracer brièvement ces évolutions.

Jusque vers les années 70 , la priorité était donnée aux problèmes quantitatifs. Des modèles hydrauliques simplifiés étaient construits pour aider le concepteur à localiser et dimensionner les ouvrages. Le problème consistait bien souvent à vérifier que les ouvrages projetés permettaient d'assurer une pression convenable en tout point du réseau et ce, dans les conditions les plus défavorables, c'est-à-dire à l'heure de pointe d'une journée de pointe.

Ces modèles ne comportaient que l'ossature principale du réseau pour des raisons de temps d'exécution des simulations et tous les calculs effectués par les programmes informatiques de l'époque ne portaient que sur une photographie de l'état du réseau à un instant donné correspondant à un niveau de demande, des hauteurs d'eau données dans les différents réservoirs et à un état des pompes et vannes sur le réseau. Ces modèles dits statiques répondaient toutefois assez bien aux problèmes posés.

Dans les années 80 , les besoins quantitatifs étant satisfaits, l'accent fut mis sur la sécurité des systèmes d'alimentation en eau. L'objectif visé était de pouvoir continuer à satisfaire la demande même dans des circonstances exceptionnelles (provoquées par l'indisponibilité temporaire d'une ressource par suite d'une pollution industrielle, le détournement d'une grosse canalisation pour cause de travaux routiers ou autoroutiers, une panne EDF de longue durée, etc.). L'utilisation par le concepteur de modèles hydrauliques dynamiques devenait alors indispensable pour mesurer l'impact de ces scénarios sur le comportement global du réseau pendant 24 heures au plus.

En effet, le problème dans ces situations de crise ne consistait pas seulement à vérifier que la pression restait convenable en tout point du réseau mais aussi que les réserves se reconstituaient normalement au bout de 24 heures et que les transferts de volumes inter-réseaux étaient possibles tout en limitant les niveaux piézométriques au refoulement des usines afin de diminuer les risques de rupture.

En fait, les modèles hydrauliques utilisés furent au départ de simples extensions des modèles statiques, c'est-à-dire que les calculs s'enchaînaient automatiquement mais l'opérateur était obligé d'intervenir dès qu'un seuil d'asservissement ou un niveau trop haut ou trop bas dans un réservoir se trouvait franchi.

Plus tard, grâce sans doute à la puissance accrue des micro-ordinateurs, ces modèles intégrèrent pour la première fois certaines conditions d'asservissement et furent alors capables de revenir en arrière et de générer automatiquement des pas de temps intermédiaires ; ceci permit d'alléger le travail du concepteur qui se trouvait confronté à des réseaux au fonctionnement de plus en plus complexe.

Aujourd'hui, les moteurs hydrauliques les plus performants, tels que SWS de la société américaine Stoner Associates, qui fut adopté par le SEDIF et son régisseur la Compagnie Générale des Eaux (CGE) en 1992, sont capables de modéliser directement la plupart des systèmes de régulation, d'affecter des variables de contrôle à chaque groupe de pompage avec des niveaux de priorité, de calculer les coûts énergétiques, de contrôler le bon fonctionnement hydraulique des pompes, de gérer plusieurs catégories de demande avec des "profils" différents et ce, sur des modèles de réseaux de grande taille comme celui de la Banlieue de Paris.

\section{IV $\square$ LES DIFFÉRENTS TYPES DE MODÈLES}

Les outils de calcul actuels des réseaux de distribution d'eau potable permettent d'envisager la construction de 3 types de modèles répondant à des besoins bien distincts : les modèles statiques, les modèles statiques profilés et les modèles dynamiques.

Les modèles statiques (ou statiques profilés) ne contiennent aucune description des asservissements associés aux divers équipements du réseau. Ainsi, pour un équipement de type pompe qui serait asservi dynamiquement au niveau de l'eau dans un réservoir, le modèle statique ne prend en 
compte que l'information de débit (ou de profil de débit) observé. Ces types de modèles comportent donc en général une description assez simplifiée des ouvrages de pompage et de régulation.

Les modèles statiques ne peuvent être utilisés qu'en mode de calcul statique (à un instant donné) alors que les modèles statiques profilés peuvent également être utilisés en mode de calcul dynamique (succession de calculs statiques enchaînés suivant un pas de temps prédéfini par l'utilisateur).

A noter que la non prise en compte des asservissements dans les modèles statiques profilés rend incertaine l'issue de calculs dynamiques effectués avec des jeux de données autres que celles réellement observées sur le terrain. C'est pourquoi ces modèles ne sont principalement utilisés que pour répondre à deux besoins spécifiques : effectuer des calages à partir d'informations issues d'une campagne de mesures ou analyser en temps différé le comportement du réseau en injectant dans le modèle les données enregistrées par un système de télémétrie.

Les modèles statiques sont surtout utilisés pour dimensionner les canalisations de nouveaux lotissements ou résoudre des problèmes de manque de pression ou de défense incendie et pour déterminer l'état permanent initial du réseau juste avant le phénomène provoquant le régime transitoire (disjonction brutale de groupes par exemple).

A la différence des modèles statiques, les modèles dynamiques comportent une description la plus complète possible du fonctionnement des équipements du réseau et des asservissements associés. Ces types de modèle sont les plus utilisés aujourd'hui car ils permettent de bien prendre en compte les contraintes d'exploitation ainsi que les modes de fonctionnement dégradés suite à des opérations de maintenance ou à des incidents. En outre, ces modèles permettent de prédire l'évolution de la qualité de l'eau distribuée et sont de ce fait de plus en plus utilisés par les exploitants pour optimiser la gestion du système de distribution $[1,2,3]$.

Dans un premier temps, le modèle permet d'établir un premier diagnostic sur le fonctionnement du réseau et de proposer différentes solutions d'aménagement en vue d'optimiser les caractéristiques hydrodynamiques du réseau qui influencent fortement l'évolution de la qualité de l'eau mise en distribution.

L'étape suivante vise à déterminer l'évolution des paramètres descriptifs de la qualité de l'eau dans le système qui peut être assimilé à un réacteur biochimique où existent de fortes interactions avec les surfaces solides. Le taux résiduel de chlore libre, qui est l'un des premiers facteurs de maîtrise de la qualité de l'eau distribuée, est en général le premier paramètre sur lequel porte la modélisation de la qualité. Mais ce n'est pas le seul, car les modèles qualité sont aussi utilisés pour étudier la contribution des différentes sources et résoudre ainsi des problèmes de mélange d'eaux de qualités différentes.

Bien entendu, comme pour les modèles hydrauliques, pour pouvoir s'appuyer en toute confiance sur les résultats des concentrations en chlore fournies par ces simulateurs dans les diverses configurations étudiées, il s'avère indispensable de procéder au préalable au calage du modèle, c'est-à-dire à l'ajustement des cinétiques de décroissance si possible sur plusieurs saisons afin de prendre en compte les variations des paramètres physico-chimiques de l'eau mise en distribution.

\section{$V$ LES NOMBREUSES APPLICATIONS POSSIBLES DE CES MODÈLES EN CONCEPTION ET EN EXPLOITATION}

Les applications possibles de ces modèles dynamiques, où les aspects conception et exploitation sont étroitement mêlés. sont très nombreuses. Aussi nous bornerons-nous à ne citer ci-après que celles qui nous semblent les plus marquantes :

- localisation et dimensionnement de nouvelles installations intégrant l'influence des ouvrages existants et la modification des asservissements et prenant en compte différents scénarios d'incident.

- test de différentes stratégies d'exploitation du réseau. voire détermination, le cas échéant, des aménagements à réaliser afin d'optimiser, en l'absence de tout incident, I'hydraulique du système. L'objectif de cette optimisation est la réduction des temps de séjour et l'élimination des zones stagnantes de façon à favoriser le maintien de la qualité de l'eau distribuée.

- gestion des grosses interventions programmées, voire des situations de crise, c'est-à-dire détermination du ou des modes d'exploitation les plus appropriés pour garantir l'alimentation en eau en tout point du réseau tout en évitant de détériorer la qualité de l'eau,

- minimisation, en l'absence de tout incident, des coûts énergétiques grâce à une meilleure gestion des différents pompages et stockages tout en conservant le même degré de sécurité d'alimentation et la même qualité d'eau distribuée,

- détermination des stratégies de désinfection avec ou sans station de re-chloration pour maintenir le résiduel de chlore en tout point du réseau dans des limites compatibles avec le maintien de la stabilité biologique de l'eau et de la qualité gustative de l'eau,

- études de cas de pollution dans différents modes d'exploitation afin d'identifier les zones à haut risque et d'établir des procédures d'urgence pour isoler et nettoyer les zones contaminées.

\section{VI $\square$ EXEMPLES D'UTILISATION DE MODÈLES DYNAMIQUES EN RÉGIME ÉTABLI}

Nous allons illustrer ces cas d'application des modèles hydrauliques dynamiques en régime établi par deux exemples concrets vécus en Région Parisienne sur le réseau du SEDIF.

L'un concerne la détermination des stratégies de pompage à adopter à l'usine principale lors d'une période d'indisponibilité d'une conduite maîtresse, pour faire face à différents scénarios d'incidents. L'autre exemple a trait à un problème d'optimisation hydraulique sous contraintes pour une meilleure gestion des temps de séjour de l'eau dans l'un des réservoirs principaux du réseau.

\subsection{Description du réseau du SEDIF}

Le réseau du SEDIF est certainement l'un des plus importants et des plus complexes de France, voire d'Europe. II comporte plus de $8500 \mathrm{~km}$ de canalisations, environ une cinquantaine de réservoirs et de stations de pompage et distribue en moyenne chaque jour plus de 1 million de $\mathrm{m}^{3}$ à 4 millions d'habitants. 


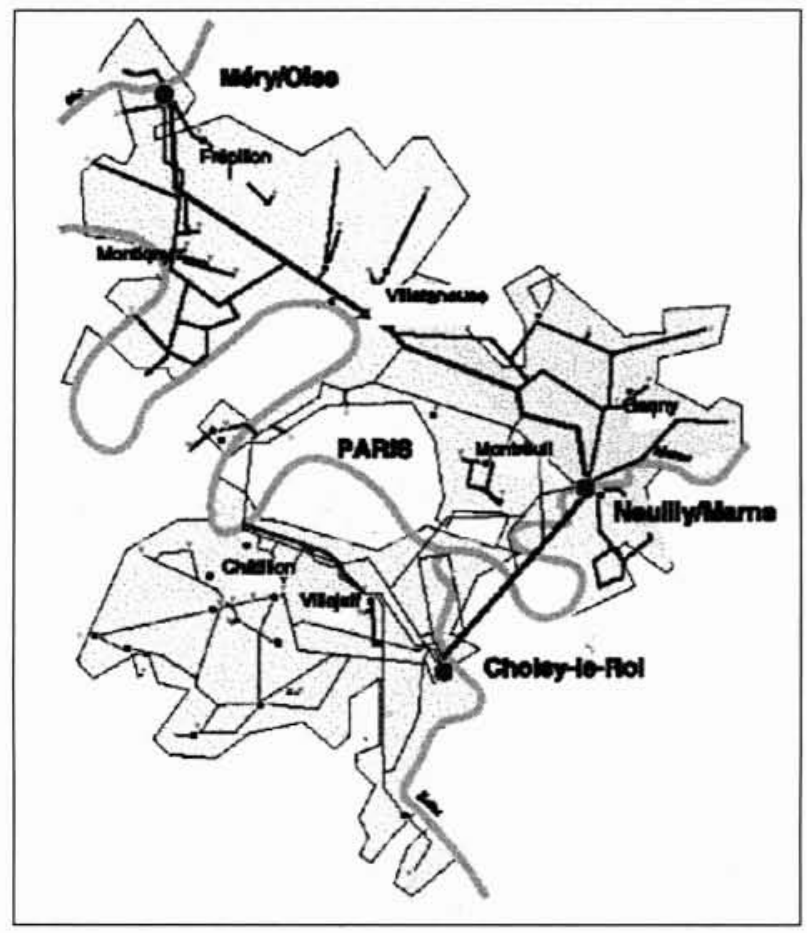

1. Le réseau du SEDIF.

Le système de distribution est divisé en 3 secteurs, chacun d'entre eux étant alimenté par une usine de traitement située près de l'une des trois rivières arrosant la Région Parisienne : Choisy-le-Roi sur la Seine, Neuilly-surMarne et Méry-sur-Oise (figure 1).

Chaque secteur comprend trois niveaux d'élévation : le niveau d'élévation le plus bas est celui alimenté directement par I'usine principale tandis que les niveaux supérieurs sont alimentés par des stations de pompage qui aspirent dans le réseau de l'étage inférieur soit directement soit par l'intermédiaire de réservoirs.

Le système d'alimentation du SEDIF comporte au final 40 zones de distribution élémentaires.

Des interconnections surpressées entre les réseaux de lère élévation des secteurs Est et Nord d'une part et Est et Sud d'autre part permettent d'assurer l'alimentation des 4 millions de franciliens même en cas de pollution prolongée de l'une des rivières.

En 1992, un département spécifique appelé Centre des Mouvements de l'Eau (CME) fut créé afin de superviser le fonctionnement de l'ensemble des ouvrages et coordonner les actions des trois secteurs d'exploitation notamment lors de grosses opérations de maintenance sur le réseau ou lors de situations de crise (pollution rivière, rupture feeder, ....). Pour remplir sa mission, le CME dispose d'un certain nombre d'outils tels que : un mini Système d'Information Géographique (SIG), un système sophistiqué de télémétrie recevant des données hydrau- liques et qualité à intervalles réguliers et un système neuronal de prévision de la consommation. Plus récemment, un simulateur de fonctionnement du réseau utilisant l'outil de modélisation SWS, fut couplé au système de télémétrie afin de permettre aux utilisateurs du CME de déterminer rapidement et avec fiabilité les mesures à prendre pour faire face aux incidents d'exploitation [4].

\subsection{Détermination des régimes de pompage à adop- ter pour faire face à différents scénarios d'inci- dents lors d'une période d'indisponibilité d'une canalisation maîtresse}

Le réseau du secteur Sud dessert 1,5 million de personnes

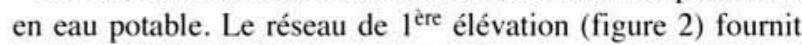
environ $50 \%$ de la demande totale du secteur Sud et est essentiellement alimenté par l'usine de traitement de Choisyle-Roi (production maximale de $800000 \mathrm{~m}^{3} / \mathrm{j}$ ) et, dans sa partie sud, par de l'eau de forage qui provient d'Arvigny (entre 30000 et $50000 \mathrm{~m}^{3} / \mathrm{j}$ ).

Il existe deux sites de stockage principaux à Châtillon $\left(155000 \mathrm{~m}^{3}\right)$ et à Villejuif $\left(53000 \mathrm{~m}^{3}\right)$ reliés à l'usine principale par une boucle composée de canalisations de diamètre $2000 \mathrm{~mm}, 1800 \mathrm{~mm}, 1500 \mathrm{~mm}$ et $1250 \mathrm{~mm}$. Trois canalisations sont connectées à cette boucle, les 2 canalisations (le $1250 \mathrm{~mm}$ inter usines et le $1250 \mathrm{~mm}$ de Joinville) assurant le secours mutuel entre les secteurs Sud et Est, et le 1500/1250 mm qui part des réservoirs de Châtillon pour desservir une longue bande étroite appelée "Le Corridor de I'Ouest" dont la consommation atteint environ $100000 \mathrm{~m}^{3} / \mathrm{j}$, soit le quart de la consommation du secteur Sud. Compte

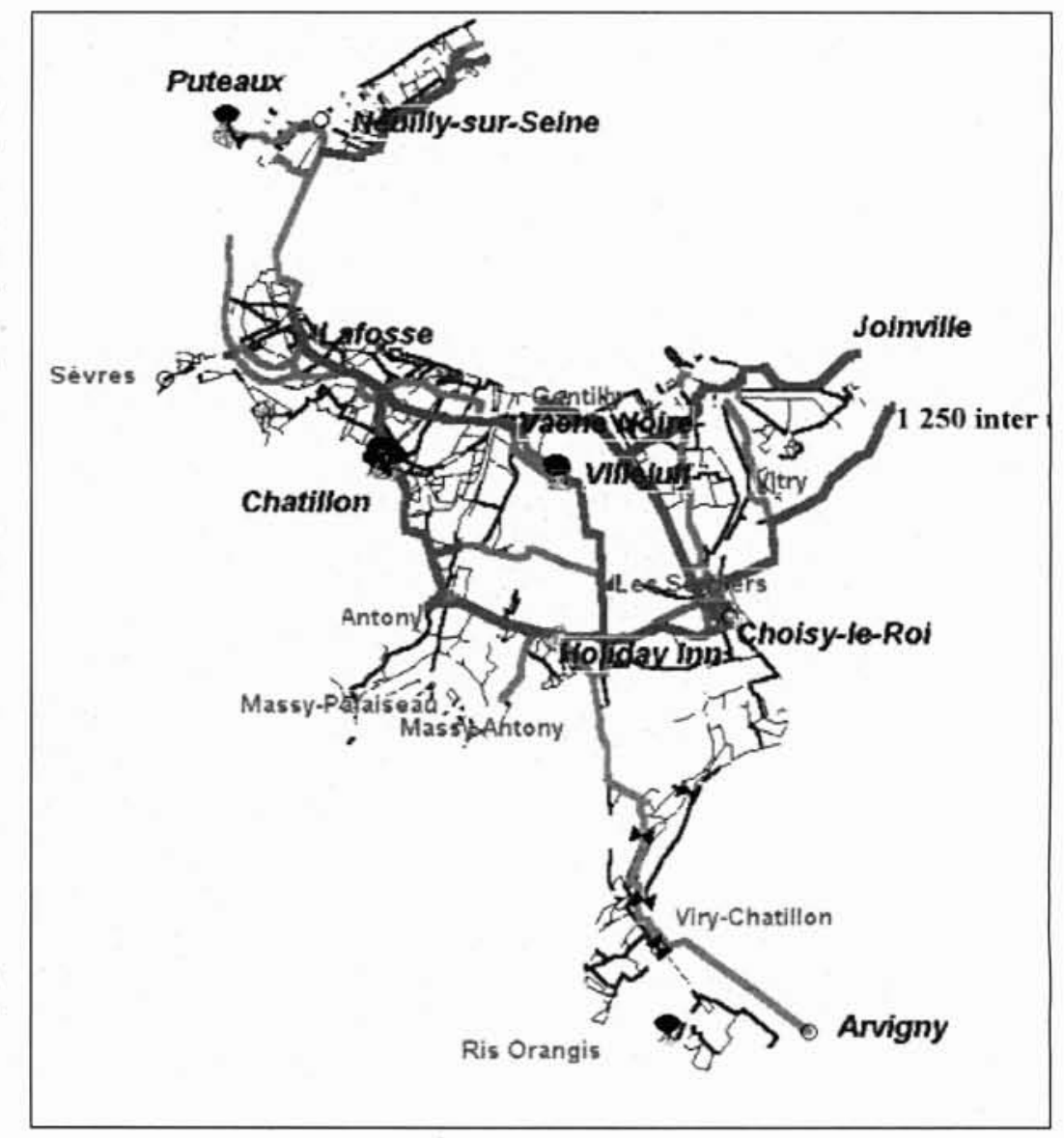

2. Modèle du réseau de 1ère élévation du secteur Sud. 


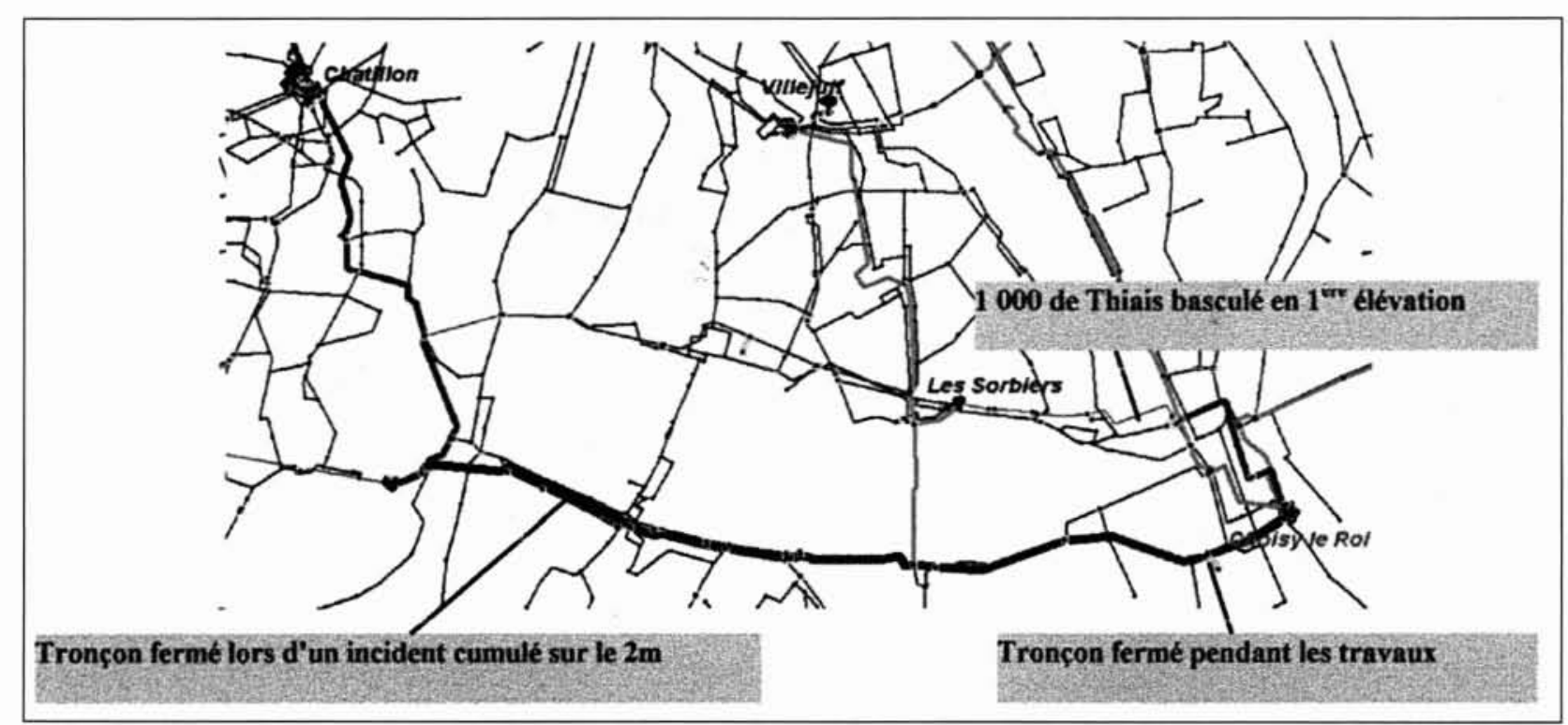

3. Situation des travaux et du 1000 de Thiais.

tenu du fait que le réservoir de Châtillon se viderait préférentiellement pour absorber la consommation, étant à un NP supérieur à celui de Villejuif, un système de vannes situé au lieu dit "La Fosse" rétablit l'équilibre hydraulique en permettant au réservoir de Villejuif d'alimenter préférentiellement le corridor de l'ouest. Enfin, le transfert d'eau vers les élévations supérieures s'effectue par l'intermédiaire des usines de pompage des Sorbiers, de Châtillon, d'Antony, de MassyPalaiseau, de Massy-Antony, de Puteaux et de Sèvres.

L'usine de Choisy-le-Roi comporte 10 pompes de caractéristiques différentes dont une, identifiée SE9, est à vitesse variable. Cette pompe offre une bonne flexibilité pour respecter les différentes consignes d'exploitation : Niveau Piézométrique (NP) maximal de 129-130 $\mathrm{mCE}$ au refoulement de l'usine, NP minimal de $90 \mathrm{mCE}$ sur le réseau, remplissage des réservoirs en fin de journée et marnage maximal des réservoirs durant la journée. Lorsque cette pompe est en révision, il faut utiliser différentes combinaisons de pompes à vitesse fixe, ce qui peut créer des à-coups de NP en sortie d'usine et conduire dans certains cas à dépasser le seuil maximum.

Au début de l'année 1996, la Ville de Choisy projetait la construction d'un gymnase sur un terrain situé non loin de l'usine et où passait la conduite principale de $2000 \mathrm{~mm}$ reliant l'usine au réservoir de Châtillon. Il fut décidé de tuber le bief situé sous le futur gymnase afin de pallier le poids de la nouvelle structure qui allait reposer sur le $2000 \mathrm{~mm}$. Ces travaux allaient nécessiter l'arrêt du $2 \mathrm{~m}$ pendant une période de 4 à 6 semaines. Or, près de la moitié de l'eau produite à l'usine de Choisy-le-Roi transite dans cette conduite. Sa fermeture allait donc modifier considérablement les caractéristiques hydrauliques du réseau avec pour conséquences prévisibles une augmentation du NP au refoulement de l'usine de Choisy et donc des risques de rupture sur le réseau et la difficulté à faire battre les réservoirs principaux.

Une analyse des mesures à mettre en œuvre pour limiter l'impact de la mise hors service ainsi qu'une bonne évaluation des conséquences hydrauliques de la non disponibilité du $2000 \mathrm{~mm}$ dans diverses conditions de consommation et divers scénarii d'incidents, furent alors menées. L'étude fut réalisée en cinq étapes, à savoir :
- développement d'un modèle hydraulique dynamique stratégique à l'aide du logiciel SWS de Stoner permettant d'analyser et de simuler le comportement actuel et futur du réseau de première élévation du secteur Sud,

- calage du modèle afin de vérifier la cohérence du comportement du modèle dans des conditions de consommation usuelle et en testant différents niveaux de production ainsi que l'impact du basculement sur le réseau de $1^{\text {ère }}$ élévation, du $1000 \mathrm{~mm}$ reliant l'usine de Choisy à la station de pompage des Sorbiers (figure 3 ),

- définition des scenarii à tester,

- utilisation du modèle pour évaluer l'impact de l'arrêt du $2000 \mathrm{~mm}$ dans les différents scenarii vis-à-vis du respect des contraintes liées aux niveaux piézométriques en réseau,

- analyse des courbes de consommation depuis 1980 pour le choix d'une période de mise hors service acceptable.

Ce sont au total 127 simulations qui ont été réalisées avec le modèle dynamique du secteur Sud correspondant à 96 scenarii avec la pompe SE9 disponible et 31 scenarii avec SE9 indisponible. Compte tenu de la durée de l'intervention, certains scenarii intégraient soit un transfert d'eau vers le secteur Est pour pallier une pollution de la Marne, soit une indisponibilité d'un feeder secondaire en plus du $2 \mathrm{~m}$. Les cas où l'usine de Neuilly-sur-Marne apporte de l'eau sur le secteur sud n'ont pas été testés car ils correspondent à une diminution de la production de Choisy donc à un NP au refoulement plus faible, dans la mesure où cet apport se fait par le surpresseur de Joinville et non par le $1250 \mathrm{~mm}$ inter-usines.

Une étude préliminaire a permis d'une part de déterminer le cas d'indisponibilité le plus critique à savoir le non-basculement du $1000 \mathrm{~mm}$ de Thiais en $1^{\text {ere }}$ élévation et d'autre part de regrouper les incidents sur "feeders" secondaires (ou incident sur un autre tronçon du $2000 \mathrm{~mm}$ ) en deux classes homogènes du point de vue de l'impact sur le NP au refoulement de Choisy ou sur les pressions dans le corridor de l'ouest.

Il est finalement apparu que le facteur limitant pour la mise hors service de longue durée du plus gros "feeder" du secteur Seine était le niveau piézométrique en sortie d'usine de Choisy. Les différentes simulations réalisées sur modèle 


\section{Mécanique des fluides}

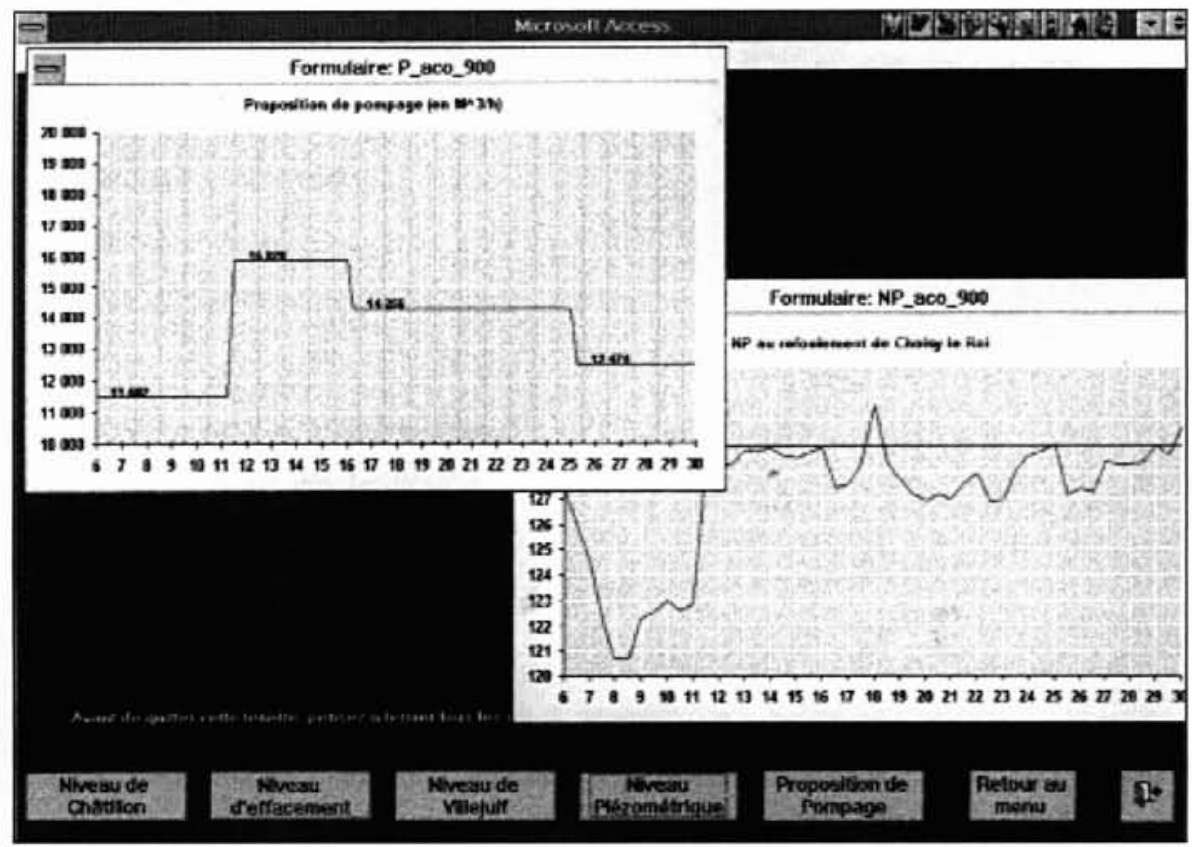

4. Exemple d'écran de l'outil

d'aide à la gestion du réseau pendant l'arrêt du $2000 \mathrm{~mm}$.

ont permis de montrer qu'il était possible d'adapter les configurations de pompage à l'usine de Choisy pour maintenir le $\mathrm{NP}$ au refoulement en dessous de la limite d'exploitation fixée pour autant que la demande ne dépasse pas de plus de $20 \%$ la moyenne annuelle.

Après avoir défini la période favorable pour une telle opération, les conséquences hydrauliques de cet arrêt ont été simulées sur modèle dans les 127 scenarii prédéfinis. En particulier, une analyse de la gestion du marnage des réserves de Villejuif et de Châtillon et des niveaux piézométriques au refoulement de l'usine de Choisy-le-Roi a permis de dégager des modes de gestion optimale de la station de pompage élévatoire de l'usine principale. Ces études ont abouti à la constitution d'un outil d'aide à la gestion du réseau qui a été déployé en salle de supervision de l'usine pendant la période d'arrêt de la canalisation de $2000 \mathrm{~mm}$ afin d'orienter quotidiennement les choix des opérateurs (figure 4).

\subsection{Optimisation hydraulique} pour la gestion des temps de séjour dans les réservoirs : cas du réservoir de Montignyles-Cormeilles

Le réseau du secteur Nord est alimenté à partir de l'usine de traitement de Méry-sur-Oise dont la capacité totale de production va bientôt atteindre $340000 \mathrm{~m}^{3} / \mathrm{j}$ avec la mise en service de la nouvelle tranche de nanofiltration. La production journalière moyenne actuelle est d'environ $170000 \mathrm{~m}^{3} / \mathrm{j}$ pour une population de 800000 habitants. Trois niveaux de desserte composent ce réseau. Le premier niveau assure l'alimentation des abonnés pour $90000 \mathrm{~m}^{3} / \mathrm{j}$ et sert au transit des besoins des élévations supérieures. Signalons, parmi les stations de reprise, celle de la nouvelle usine de pompage de Porte-Rouge qui a fait l'objet d'une étude anti-bélier très complète (cf. chapitre VII).

Le réservoir d'équilibre principal de cette zone est celui de Montigny R3. Comme les autres réservoirs des réseaux de

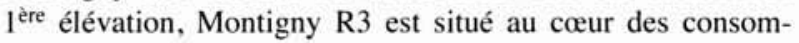
mations à desservir et fonctionne en mode dérivation. Cela signifie que le réservoir est "branché" sur le réseau par des conduites d'alimentation et de distribution qui ne sont pas dissociées et que de ce fait, une partie plus ou moins importante du débit refoulé par l'usine principale de Méry-sur-Oise ne transite pas par le réservoir et ne participe donc pas au renouvellement de l'eau dans celui-ci.

La mise en série du réservoir de Montigny R3 vise donc à réduire sensiblement les temps de séjour de l'eau dans cet ouvrage et par conséquent à optimiser la désinfection en réseau. Cette mise en série était techniquement possible par une réorganisation du réseau de $1^{\text {ère }}$ élévation (fermeture d'une

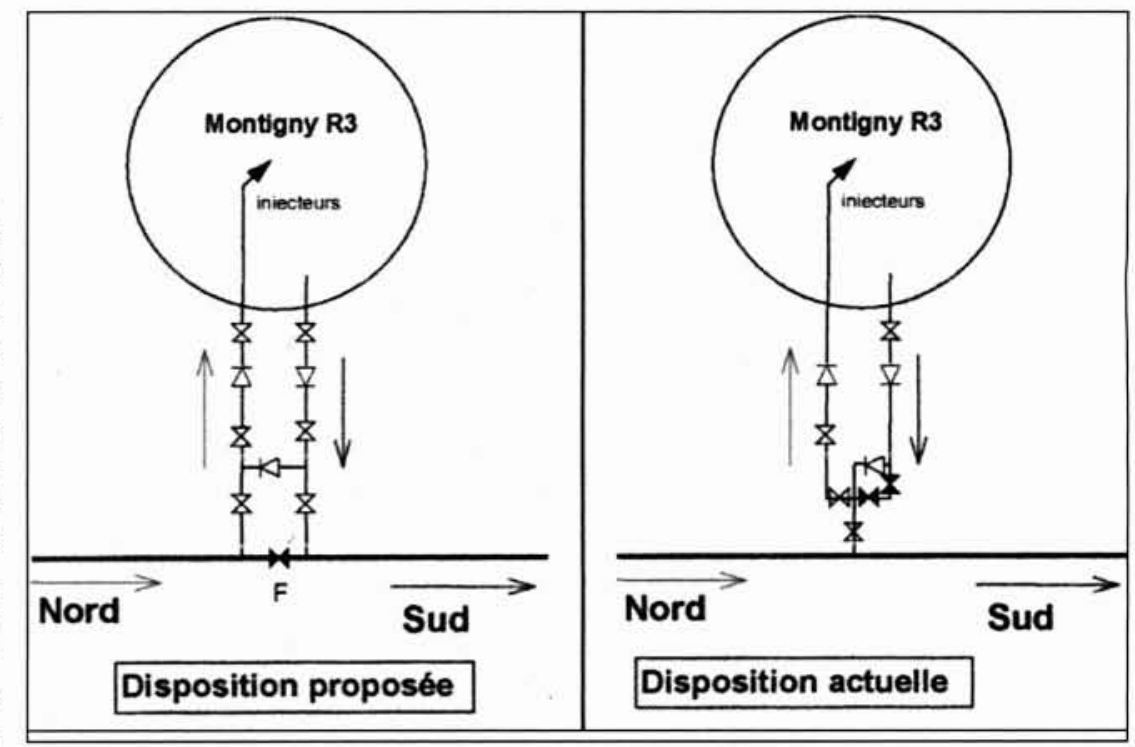

5. Dispositions actuelle et projetée du réservoir de Montigny. 


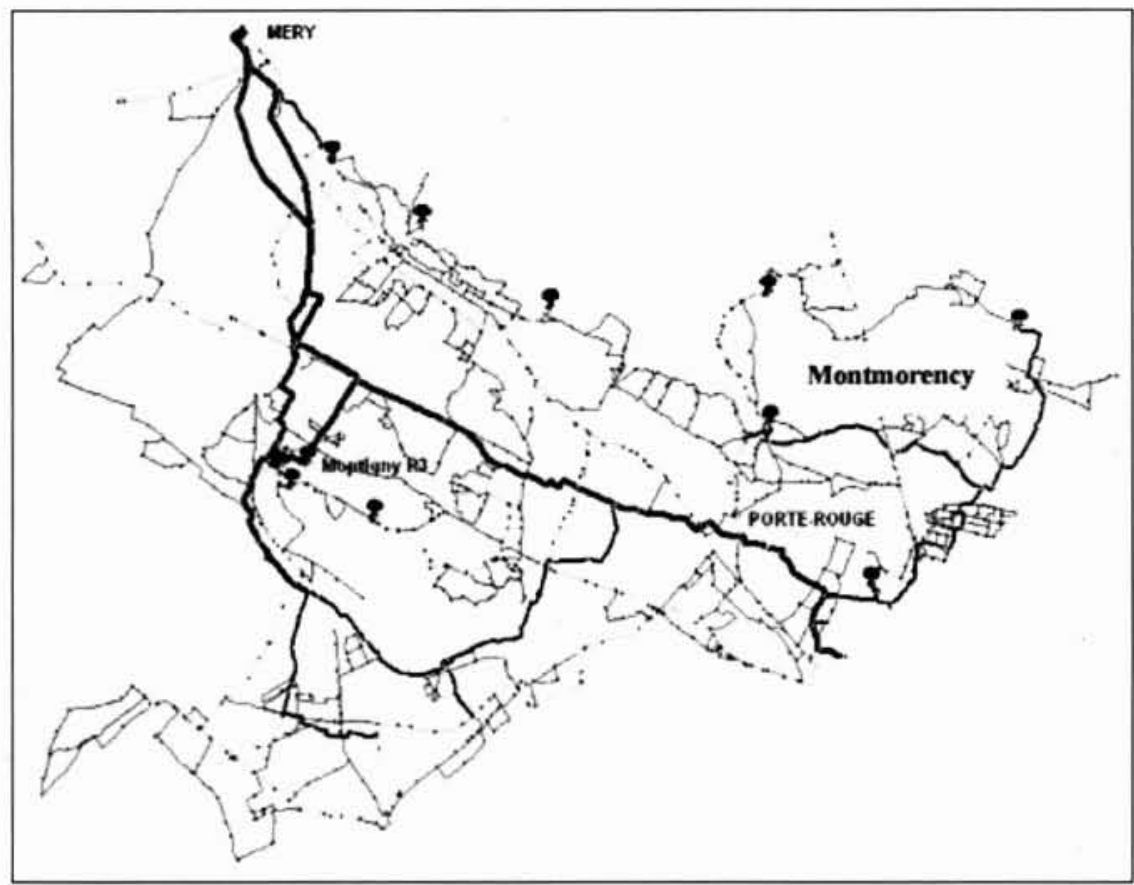

6. Le modèle du réseau du secteur Nord.

vanne sur une conduite de $1000 \mathrm{~mm}$ afin de forcer la circulation dans un sens) et un aménagement local des conduites d'alimentation et de distribution du réservoir (figure 5).

Mais une telle opération qui s'inscrivait dans un contexte plus global d'une adaptation importante de la gestion du réseau du secteur Nord (consécutive à la mise en service, d'ici à la fin du siècle, d'une tranche de nanofiltration à l'usine de Méry/Oise et de l'exploitation du nouveau réservoir de Montmorency), pouvait affecter fortement le comportement hydraulique du réseau.

Des modèles hydrauliques dynamiques intégrant les différents aménagements à venir ont donc été développés avec le logiciel SWS (figure 6) afin d'analyser les conséquences de cette mise en série sur le niveau piézométrique au refoulement de l'usine de Méry-sur-Oise et plus particulièrement sur l'axe entre Méry et Montigny, sur la gestion des réserves et sur la gestion du pompage de $1^{\text {ère }}$ élévation.

L'étude sur modèle a démontré que le mode de fonctionnement en série permettait, suivant le niveau de production de l'usine, de diviser par 3 ou 4 le temps de séjour actuel de l'eau au sein du réservoir de Montigny R3 (figure 7). En moyenne (production de $170000 \mathrm{~m}^{3 / \mathrm{j}}$ ), le temps de séjour est ramené de 65 heures à 18 heures et ce, sans modification importante dans la gestion du pompage élévatoire de l'usine de Méry (simple ajustement des vitesses de rotation des groupes à vitesse variable).

Elle a également montré qu'il était possible de maintenir un niveau plus élevé des réserves mais au prix d'une gestion légèrement plus séquencée des groupes élévatoires de l'usine de Méry et donc avec un impact non négligeable sur la gestion des réserves d'effacement de l'usine.

Plusieurs options de mise en série ont pu être testées correspondant à des configurations de réseau avec ou sans renforcement. Une analyse économique a fait clairement ressortir la supériorité de la solution sans renforcement, le gain énergétique engendré par les solutions avec renforcement du réseau n'étant pas en rapport avec le montant des investissements induits.

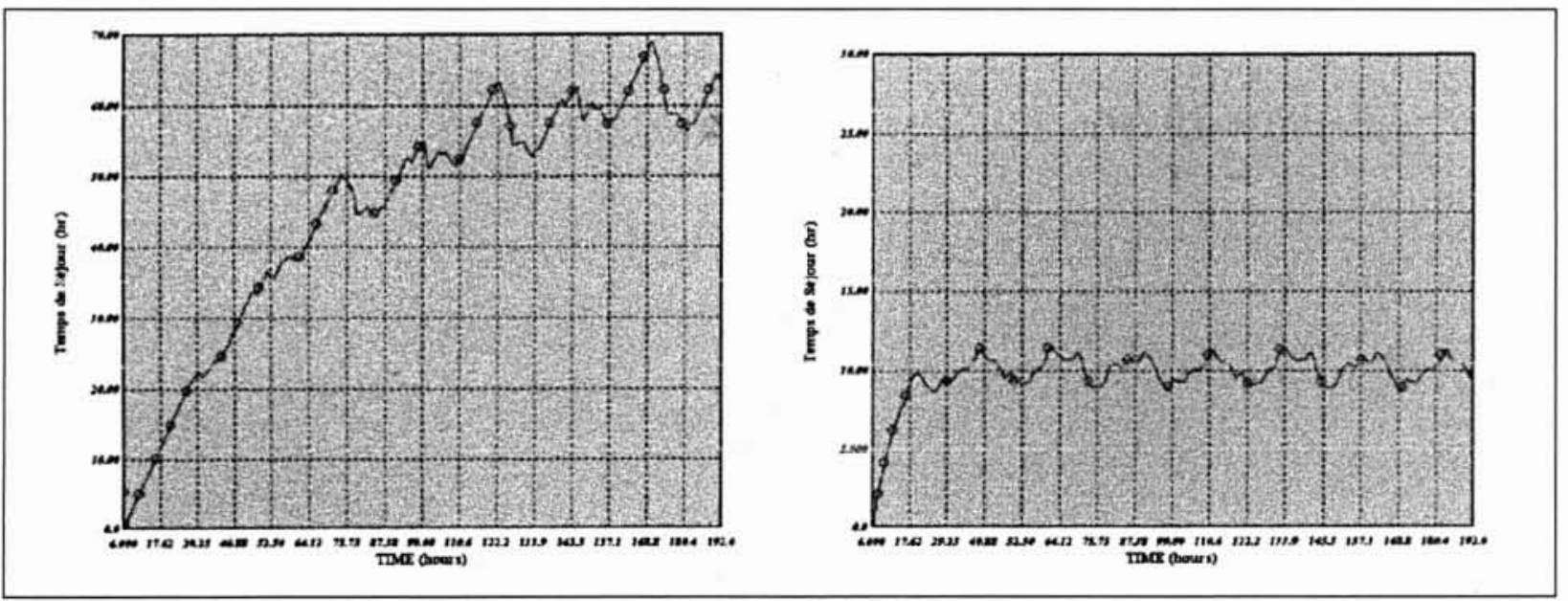

7. Temps de séjour de l'eau au sein du réservoir avant et après la mise en série. 


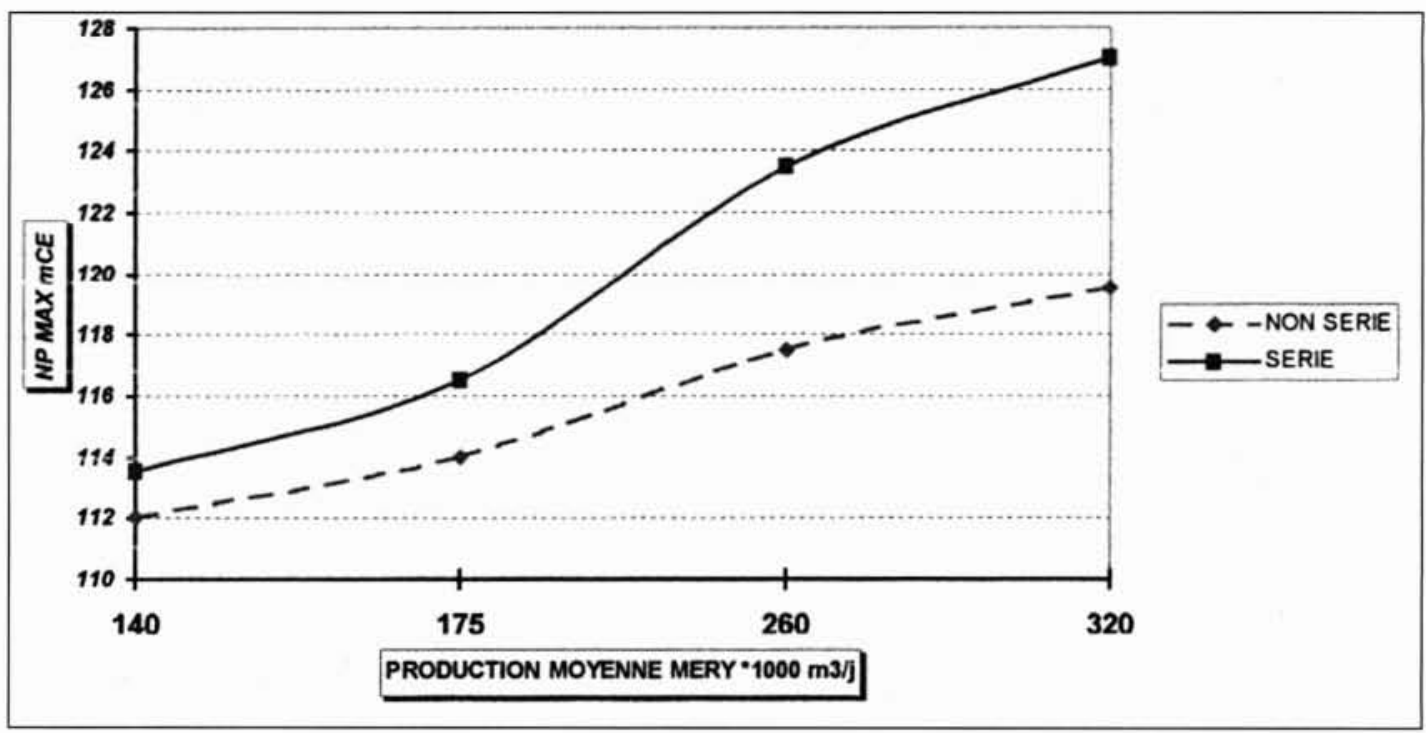

8. NP maximal en fonction du niveau de production de Méry.

L'étude sur modèle a permis de tracer l'évolution du niveau piézométrique maximal en fonction du niveau de production de l'usine de Méry dans les configurations actuelle et future du réservoir de Montigny R3 (figure 8).

Compte tenu de la réduction naturelle des temps de séjour de l'eau dans le réservoir dans sa disposition "en dérivation" lorsque le niveau de production augmente, il a été décidé, afin de limiter les conséquences d'une hausse du NP trop importante au refoulement de Méry, d'adopter deux modes d'exploitation : jusqu'à un niveau de production de Méry de $200000 \mathrm{~m}^{3} / \mathrm{j}$, le réseau de $1^{\text {ère }}$ élévation du secteur Nord est géré avec le réservoir de Montigny R3 configuré en mode série et au-delà, le mode de fonctionnement ancien, réservoir en dérivation, est privilégié. Cela suppose une automatisation fiable des vannes assurant le passage d'une configuration série à une configuration non-série et inversement.

\section{VII — EXEMPLE D'UTILISATION DE MODÈLE EN RÉGIME TRANSI- TOIRE : DÉTERMINATION DE LA PROTECTION ANTIBÉLIER DE LA STATION DE PORTE-ROUGE}

Dans le cadre de la réorganisation des réseaux de $2^{\text {ème }}$ élévation de Porte-Rouge et de Pierrefitte desservis par le nouveau réservoir de Montmorency et la mise en œuvre de la $2^{\text {ème }}$ liaison de secours entre les secteurs Nord et Est, l'usine de Porte-Rouge, dont les équipements hydrauliques étaient par ailleurs vétustes, devait être complètement refondue. La capacité nominale de la nouvelle usine devait être portée à $90000 \mathrm{~m}^{3} / \mathrm{j}$ (contre $17000 \mathrm{~m}^{3} / \mathrm{j}$ pour l'ancienne). Cette usine a la particularité d'aspirer directement dans le réseau de $1^{\text {ère }}$ élévation du secteur Nord et donc d'induire, en l'absence de toute protection, des surpressions importantes sur le réseau amont en cas de disjonction des groupes par suite d'une coupure EDF.

Une première étude sur la protection anti-bélier de la future usine de Porte-Rouge avait conclu à l'inutilité d'un clapet by-pass d'une part et à la nécessité d'installer un volume total de ballon de $287 \mathrm{~m}^{3}$ à l'aspiration et de $47 \mathrm{~m}^{3}$ au refoulement d'autre part. Ces volumes avaient été établis dans les conditions suivantes :

- débit maximal refoulé dans les conditions les plus défavorables de $105000 \mathrm{~m}^{3} / \mathrm{j}$ au moment de la disjonction, pression résiduelle au refoulement sur un profil de canalisation théorique : $5 \mathrm{mCE}$,

- NP maximal admissible à l'aspiration : $125 \mathrm{mCE}$,

- liaison actuelle Eaubonne - Porte Rouge en $1250 \mathrm{~mm}$ (renforcement prévu dans le cadre de la mise en œuvre de la liaison Nord, $2^{\text {ème }}$ interconnection de secours entre les secteurs Nord et Est).

La superficie du terrain disponible ne permettant pas d'implanter une telle capacité de ballon anti-bélier, il avait été envisagé, compte tenu du fait que les groupes projetés étaient à vitesse variable, d'utiliser des volants d'inertie comme moyen de protection. Mais la masse très importante des volants, nécessaire pour atteindre un niveau de protection identique à celui procuré par les ballons anti-bélier, risquait d'engendrer d'autres types de contraintes (vibrations, bruit, résistance de la structure).

La solution volant d'inertie n'étant pas souhaitable, l'étude a donc été reprise avec l'objectif de minimiser le volume de ballon anti-bélier, notamment en mixant plusieurs types de protection. Par ailleurs, cette nouvelle étude a dû tenir compte du profil réel de la canalisation de refoulement de Porte-Rouge et des différentes phases dans la réalisation de l'usine et dans l'aménagement du réseau d'aspiration de première élévation.

- Phase 1: Le réseau est dans sa configuration actuelle. L'usine alimente le nouveau réseau de Montmorency (consommation moyenne de $35000 \mathrm{~m}^{3} / \mathrm{j}$ ). Le dimensionnement des ouvrages se faisant au coefficient journalier de 1,4, l'étude de la disjonction est réalisée pour deux groupes fonctionnant à plein régime.

- Phase 2: On envisage dans la configuration actuelle du réseau de faire fonctionner la liaison Nord (liaison de secours inter-secteurs complémentaire à celle de Villetaneuse) : c'est donc la disjonction de trois groupes à débit maximum qui est étudiée. 


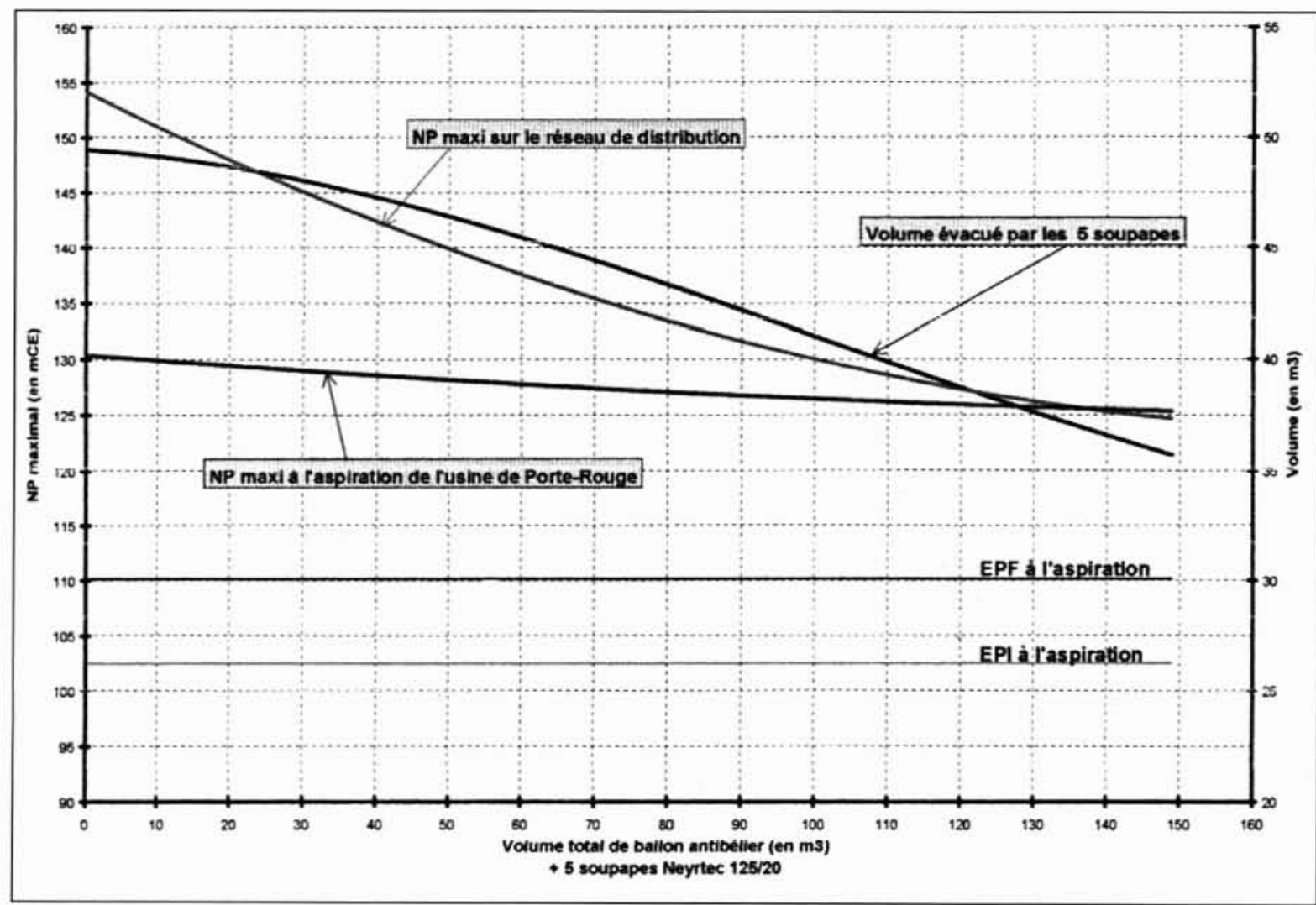

9. NP maximal en fonction du volume antibélier en phase 4.

- Phase 3 : Idem phase 2 plus renforcement de la conduite d'aspiration de l'usine en la doublant par un $600 \mathrm{~mm}$.

- Phase 4 : Idem phase 3 plus renforcement du réseau amont par la pose d'un $800 \mathrm{~mm}$ sur l'axe Eaubonne - Porte Rouge.

\subsection{Protection à l'aspiration par mixage d'équipements anti-bélier}

Une solution classique consiste à ajouter des soupapes de décharge à l'aspiration de l'usine de façon à réduire le volume total d'anti-bélier nécessaire. La figure 9 montre le niveau de protection obtenu sur le réseau de distribution (courbe bleue) et à l'aspiration de l'usine de Porte-Rouge (courbe rouge) en fonction du volume anti-bélier et avec 5 soupapes (la courbe noire donne le volume évacué) en phase finale d'aménagement.

Le tableau 1 récapitule les résultats obtenus pour deux niveaux piézométriques limites : 125 et $130 \mathrm{mCE}$, atteints à l'aspiration de Porte-Rouge et sur le réseau de distribution.

On peut noter d'une part que le renforcement du réseau de

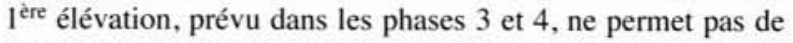
diminuer le volume anti-bélier nécessaire et d'autre part qu'il n'est pas possible de protéger l'aspiration de Porte-Rouge endeça de $125 \mathrm{mCE}$, qui constitue une valeur asymptotique.

\section{- 7.2 Protection au refoulement}

La protection anti-bélier par volant d'inertie ne pouvant pas être retenue, le refoulement de l'usine doit posséder son propre moyen de défense contre la dépression. L'aspiration et le refoulement n'ayant plus de lien hydraulique après disjonction de l'usine, les résultats de la 1 ère étude ont été réactualisés pour tenir compte des modifications du profil de la canalisation reliant Porte-Rouge à Montmorency.

Afin de minimiser le volume anti-bélier, le niveau de sécurité a été réduit (pression résiduelle de $1 \mathrm{mCE}$ dans les conditions les plus extrêmes), ce qui permet par ailleurs de

Tableau I. - Résultats obtenus dans chaque phase pour deux niveaux piézométriques limites.

\begin{tabular}{|c|c|c|c|c|c|c|c|c|}
\hline & \multicolumn{4}{|c|}{ NP maximal $=125 \mathrm{mCE}$} & \multicolumn{4}{|c|}{$\mathrm{NP}$ maximal $=130 \mathrm{mCE}$} \\
\hline & $\begin{array}{c}\text { Volume total } \\
\mathrm{AB}(\mathrm{m} 3)\end{array}$ & $\begin{array}{l}\text { Volume } \\
\text { air (m3) }\end{array}$ & $\begin{array}{l}\text { NB de } \\
\text { soupapes }\end{array}$ & $\begin{array}{c}\text { Volume } \\
\text { évacué }(\mathrm{m} 3)\end{array}$ & \begin{tabular}{|c|} 
Volume total \\
$\mathrm{AB}(\mathrm{m} 3)$
\end{tabular} & $\begin{array}{c}\text { Volume } \\
\text { air (m3) }\end{array}$ & $\begin{array}{c}\text { NB de } \\
\text { soupapes }\end{array}$ & $\begin{array}{c}\text { Volume } \\
\text { évacué }(\mathrm{m} 3) \\
\end{array}$ \\
\hline$=1$ & 115 & 74 & 4 & 23 & 80 & 46 & 4 & 30 \\
\hline PHASE 2 & 145 & 80 & 5 & 31 & 110 & 54 & 5 & 37 \\
\hline PHA & $140^{*}$ & 125 & 5 & 37 & 95 & 72 & 5 & 44 \\
\hline PHASE 4 & $140^{*}$ & 125 & 5 & 36 & 100 & 85 & 5 & 42 \\
\hline
\end{tabular}

- NP max légérement supérieur à 125 mCE (valeur asymptotique) 


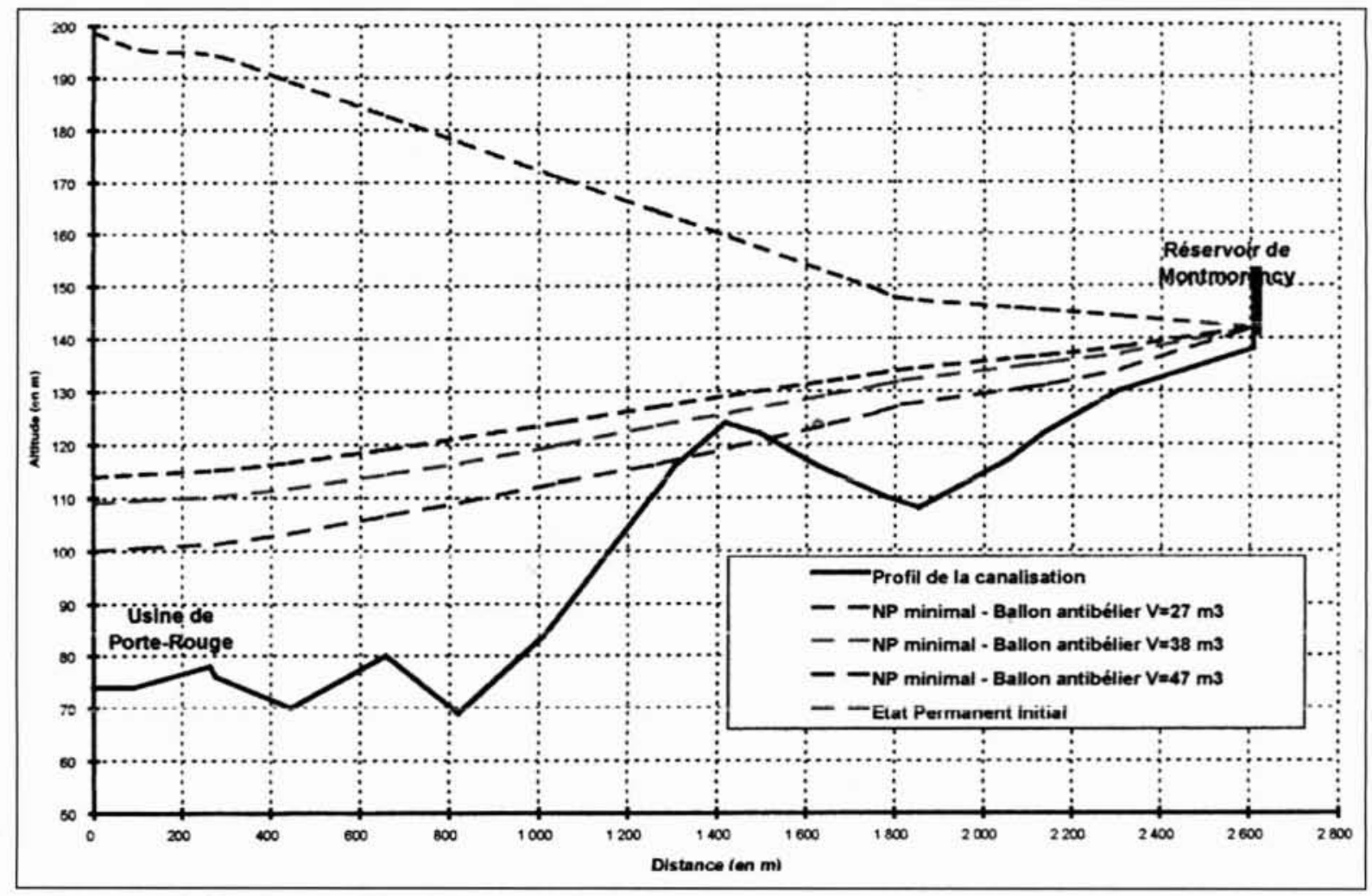

10. Courbes de NP minimal au refoulement en fonction du volume du ballon antibélier.

"gommer" l'impact du profil réel de la conduite de refoulement qui se trouve être plus défavorable que le profil théorique initial. La figure 10 donne la valeur, pour différents volumes de ballon anti-bélier, du niveau piézométrique minimal engendrée par la disjonction de l'usine en phase finale d'aménagement. Un ballon anti-bélier d'un volume minimal de $38 \mathrm{~m}^{3}$ dont $50 \%$ d'air suffit à garantir une pression minimale de $1 \mathrm{mCE}$ en tout point du refoulement.

En conclusion, l'étude en régime transitoire effectuée dans le cadre de la réalisation de la future usine de Porte-Rouge qui aspire directement sur le réseau de lère élévation du secteur Nord, a permis de déterminer les moyens de protection anti-bélier optimaux à installer à terme pour respecter les contraintes de niveau piézométrique maximal à l'aspiration (125-126 mCE) et minimal au refoulement (pression résiduelle de $1 \mathrm{mCE}$ au point le plus haut). Ces moyens optimaux sont :

\section{A l'aspiration}

-2 ou 3 ballons anti-bélier de $140 \mathrm{~m}^{3}$ de volume total dont $125 \mathrm{~m}^{3}$ d'air sous une pression de 4 bars,
- 5 soupapes NEYRTEC type 125/20 réglées à un niveau d'étanchéité de $120 \mathrm{mCE}$,

- Une bâche tampon de $36 \mathrm{~m}^{3}$.

Au refoulement

- Un ballon anti-bélier de $38 \mathrm{~m}^{3}$.

\section{Bibliographie}

[1] Vibert, A., Menge, C., Schulte, A.M. \& El.Ain, C. - Water quality Modeling Case Studies in Paris, France. Presented at the AWWA Computer Conference, Los Angeles, California (1994).

[2] Gagnon, J.L. \& T. Bowen, P. - Supply Safety and Quality of distributed Water, a contradiction overcome by the use of high performance models. Presented at the AWWA Computer Conference, Chicago, IIlinois (1996).

[3] Gagnon, J.L. - Chlorine modeling case study for the Seine network located in the Paris suburbs area. Presented at the IWSA Congress. Madrid, Spain (1997).

[4] Bonema, S.R., Elain, C., Mercier, M. \& Tiburce, V. - Linking SCADA with Network Simulation. Presented at the AWWA Annual Conference, Anaheim, California (1995). 